\title{
Bacteriological Profile of Urinary Tract Infection in Infants
}

\author{
Neha Shah*, Sachin Wankhede and Arvind V. Bhore \\ Department of Microbiology, Smth Kashibai Navale Medical College and General Hospital, \\ Pune, Maharashtra, India \\ *Corresponding author
}

\begin{tabular}{|l|}
\hline K e y w o r d s \\
$\begin{array}{l}\text { Urinary tract infection, } \\
\text { Bacteriological profile of } \\
\text { UTI, Drug sensitivity In } \\
\text { UTI }\end{array}$ \\
\hline Article Info \\
\hline $\begin{array}{l}\text { Accepted: } \\
\text { 20 March } 2018 \\
\text { Available Online: } \\
\text { 10 April } 2018\end{array}$ \\
\hline
\end{tabular}

\section{Introduction}

Urinary tract infection (UTI) is an important cause of morbidity in infants. It is mainly due to the ascending infection from urethra (Jitendranath et al., 2015) Principal risk factors include immature immunity,

\section{A B S T R A C T}

Urinary tract infection (UTI) is an important cause of morbidity in infants. The incidence of UTI is more common among boys. Inappropriately treated cases of UTI can further lead to major acute complications such as cystitis, pyelonephritis, bacteremia. Timely diagnosis and treatment is of paramount importance to prevent rare but serious complications. The objective of this study is to isolate and identify the organisms causing UTI in infants and to study antibiotic susceptibility pattern of the isolated organisms. In this cross-sectional prospective study, 480 infants with suspected of urinary tract infection, were evaluated at Smth Kashibai Navale medical college and general hospital between October 2016 to September 2017. Urine was collected by clean catch mid-stream technique or suprapubic aspiration and sent for culture sensitivity. After identifying bacteria, antimicrobial susceptibility testing was performed by the Kirby-Bauer disc diffusion method. The results were interpreted according to the Clinical and Laboratory Standards Institute (CLSI) guidelines 2013. Most common symptom was fever with suspected UTI. Of 480 UTI suspected cases 192(40\%) was culture positive. In present study there was male preponderance. Majority of the isolates were gram negative bacilli followed by gram positive cocci. The most common organism was E. coli, Enterococcus Klebsiella species. Majority of gram negative organisms were sensitive to amikacin, nitrofurantoin, gentamicin and imipenem while gram positive cocci were sensitive to nitrofurantoin, vancomycin, and teicoplanin. Overall susceptibility test showed the highest resistance to ampicillin and cotrimaxazole (TMP-SMX). Urinary tract infection is common in infants and it varies with age and gender. Appropriate evaluation of infants with history, physical examination, urine analysis and microbiological testing of urine should be mandatory. This study highlights the better efficacy of nitrofurantoin and aminoglycosides, imipenam and can be included as empirical therapy. 
Inappropriately treated cases of UTI can further lead to major acute complications such as cystitis, pyelonephritis, bacteremia (Vernon et al., 1997). Timely diagnosis and treatment is of paramount importance to prevent rare but serious complications such as renal scarring, impaired renal growth, recurrent pyelonephritis, impaired glomerular filtration, hypertension, end stage renal disease (Butler et al., 2015). If not diagnosed and managed properly recurrence is common (15-20\%), especially in the first year after an initial occurrence and the risk increases with the number of prior occurrences (Foxman, 2014). However establishing a diagnosis in infants is challenging; as they are pre-verbal and also collecting uncontaminated urine is difficult. (Mori et al., 2007)

The classic signs of UTI such as frequency, urgency, dysuria, flank, and supra-pubic pain in older children are not present in infants. Fever is most common symptom (Winberg et al., 1974). So to establish diagnosis and for proper treatment microbiological evaluation of urine and antibiotic sensitivity is mandatory while planning treatment. The objective of this study is to isolate and identify the organisms causing UTI in infants and to study antibiotic susceptibility pattern of the isolated organisms.

\section{Materials and Methods}

In this cross-sectional prospective study, 480 infants with suspect of urinary tract infection, were evaluated in Smth Kashibai Navale medical college and general hospital between October 2016 to September 2017. Patients who were referred to hospital pediatric emergency room and those presenting to paediatric outpatient department (OPD) were considered outpatients, and patients who were admitted to the ward or intensive care unit (ICU) and developed UTI at or after 3 days of admission, were considered inpatients with nosocomial infections. Infants presenting with symptoms of UTI or only fever with suspected UTI were included. Patients who have received antibiotics before urine culture, those who have treated empirically before presenting to us, samples that grew more than 1 type of micro-organism and fungal infection were excluded from the study.

Urine was collected by clean catch mid-stream technique or suprapubic aspiration and sent for culture sensitivity. Samples were cultured on blood agar and MacConkey agar by using semiquantitative method of culture. Diagnosis of UTI was made by means of positive urine culture (colony count $>$ or $=100000 \mathrm{CFU} / \mathrm{ml}$ of urine (Ismaili et al., 2011). Organisms were identified by morphology and biochemical reactions.

After identifying bacteria, antimicrobial susceptibility testing was performed by the Kirby-Bauer disc diffusion method. The results were interpreted according to the Clinical and Laboratory Standards Institute (CLSI) guidelines 2013 (Budd et al., 2013). Susceptibility of gram-negative bacteria was tested against disks of nitrofurantoin, nalidixic acid, ampicillin, colistin, cefotaxime, imipenem, piperacillin, ciprofloxacin, chloramphenicol, gentamicin, amikacin, ampicillin- sulbactam, cotrimoxazol, tetracycline, tobramycin. Disks used for grampositive bacteria included: nitrofurantoin, nalidixic acid, ampicillin, cefotaxime, imipenem, ciprofloxacin, chloramphenicol, gentamicin, trimethoprim/sulfamethoxazole (TMP-SMX), tetracycline, vancomycin, linezolid, cefoxitin, cloxacillin, penicillin.

Results were recorded includes patient's demographic profile, hospitalization status, previous treatment, microbiology laboratory data which includes type of organism and its antibiotic sensitivity. 


\section{Ethical consideration}

All these samples were a part of routine diagnosis, so ethical consideration was not necessary.

\section{Results and Discussion}

Most common symptom was fever with suspected UTI followed by suprapubic pain, frequency and dysuria (crying while micturation). Of 480 UTI suspected cases 192(40\%) was culture positive. Among these culture positive subjects $111(58.3 \%)$ were boys and 81 (42.6) \% were girls. Majority of the isolates were gram negative bacilli $119(62 \%)$ followed by gram positive cocci 73(38\%). The most common organism was E. coli 77(40\%) followed by Enterococcus 52 (27\%), Klebsiella species 25(13\%), Staphylococcus species 21(11\%) Pseudomonas species 11(6\%), and Proteus species 6(3\%) (Figure 1).

Antibiotic sensitivity: Majority of gram negative organisms were sensitive to amikacin, nitrofurantoin, gentamicin and imipenem while gram positive cocci were sensitive to nitrofurantoin, vancomycin, and teicoplanin. Overall susceptibility test showed the highest resistance to ampicillin (82.3\%) and cotrimaxazole (TMP-SMX) (77.3\%).

Antibiotic susceptibility pattern of most common pathogen i.e. E. coli shows that it was $100 \%$ sensitive to aminoglycosides (amikacin, gentamicin), nitrofurantoin and imipenam. It also had good sensitivity $94 \%$ to cefotaxim; while it was less susceptible to ampicillin and cotrimaxazole. Antibiotic susceptibility pattern of Klesialla was also similar to E. coli. It was $100 \%$ sensitive to aminogycosides and imipenam and less to ampicillin and cotrimaxzole (Table 1). Enterococci were $100 \%$ susceptible to vancomycin, ticoplanin and nitrofurantoin and to lesser degree to erythromycin (74\%), doxycyclin (60\%).

UTI, one of the most frequently occurring bacterial infections in infants and is a serious global health problem affecting millions of infants every year (Spencer et al., 2010). Majority of infants present with febrile illness with no other obvious symptom of UTI (Downs S., 1999). In the present study also most common presentation of UTI was febrile illness. This is mainly due to pre-verbal phase of infants leading to communication barrier. Prevalence of UTI varies with age and sex with some studies showing female preponderance (Elder J., 2007) while in some it is male preponderance (Winberg et al., 1974) while few has shown UTI occurs equally in boys and girls in infancy (Yilmaz et al., 2016). In the present study boys were more commonly affected than girls.

Majority of UTIs are caused by Gram negative organisms. Worldwide most common organism isolated is E. coli (Winberg et al., 1974; Elder, 2007). Similar to this our study also shows $E$. coli being the most common uropathogen representing $40 \%$ of all culture positive cases. In this present study second most common pathogen was Enterococcus (27\%) followed by Klebsiella (25\%). Literature varies with respect to other pathogens such as Klebsiella, Enterococci; few shows Klebsiella being second common pathogen leading to UTI in infants (Rai et al., 2008). Gram positive cocci such as enterococci are also one of the common organism leading to UTI in infants, Gupta et al., confirms similar finding with our study (Gupta et al., 2015).

Antibiotic susceptibility testing is the backbone for managing UTI in infants. Empirical therapy, over the counter antibiotic prescriptions has shown to increase drug resistant pathogens. 
Table.1 Antibiotic susceptibility of E. coli and Klebsiella

\begin{tabular}{|l|l|l|}
\hline Antibiotic & E.coli & Klebsiella \\
\hline Amikacin & $100 \%$ & $100 \%$ \\
\hline Nitrofurintoin & $100 \%$ & $100 \%$ \\
\hline Gentamicin & $100 \%$ & $100 \%$ \\
\hline Imepenam & $100 \%$ & $100 \%$ \\
\hline Norfloxacin & $70 \%$ & $60 \%$ \\
\hline Cefotaxim & $94 \%$ & $84 \%$ \\
\hline Ampicillin & $10 \%$ & $8 \%$ \\
\hline Cotrimaxazole & $33 \%$ & $28 \%$ \\
\hline
\end{tabular}

Fig.1 Distribution of organisms isolated

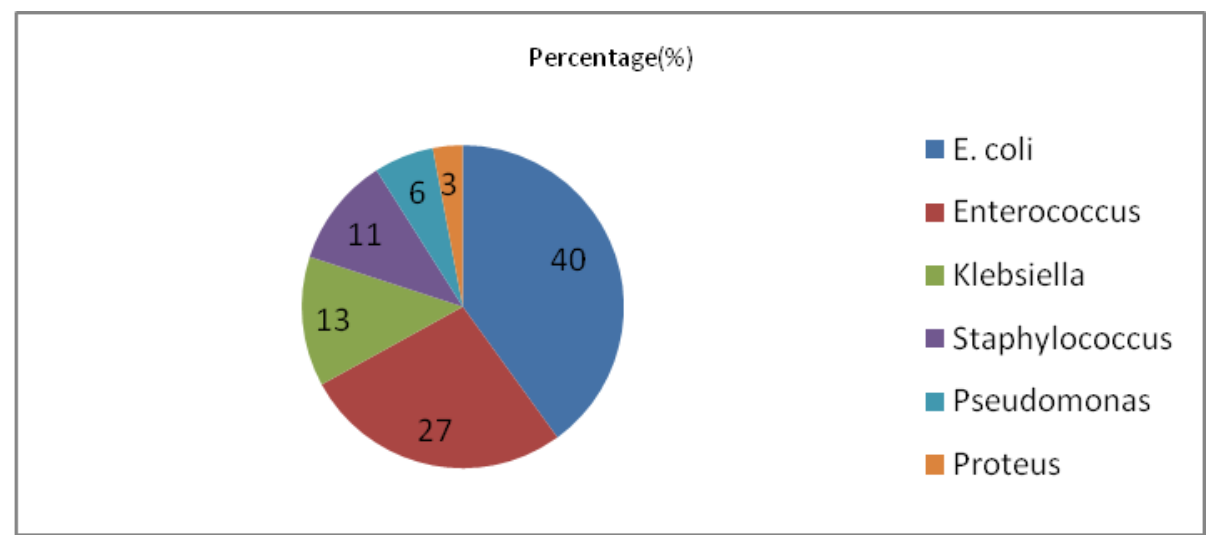

This is particularly true for developing countries like India where there are no specific guidelines and control over prescribing antibiotics (Patel et al., 2014).

In the present study E. coli was the most common uropathogen. It was $100 \%$ sensitive to aminoglycosides such as amikacin, gentamicin. Sensitivity to Imepenam and Nitrofurontoin was also $100 \%$. Literature also supports similar findings; majority studies shows that E. coli are most sensitive to aminoglycosides, nitrofurintoin and carbepenam group of drugs (i.e. imipenam) (Butler et al., 2015; Downs S., 1999). In present study $70 \%$ of $E$. coli isolates were sensitive to fluroquinolones but various Studies from other part of India have shown variable pattern of sensitivity of $E$. coli to fluroqunolones like norfloxacin (Taneja et al.,
2004; Gould et al., 2009). Cephalosporins are one of the common antibiotics used empirically in treatment of fever with suspected UTIs. Present study shows that $E$. coli has good sensitivity to cefotaxim (94\%) but rest of the literature shows increasing trend of drug resistance to cephalosporins (Salles, 2013). Bryce et al., in their systemic review have found nosocomial UTIs with $E$. coli are more resistant to cephalosporins (Bryce et al., 2016).

In the present study maximum $E$. coli isolates were resistant to ampicillin (90\%) followed by cotrimaxzole $(77 \%)$. These findings are consistent with overall trend in India [Gupta et al., 2015; Taneja et al., 2004) as well as other part of world (Gould et al., 2009) as its well-known fact that poor gram negative coverage with penicillin group of antibiotics. 
Klebsiella was the second common gram negative organism in the present study. Its antibiotic sensitivity and resistance pattern is more or less similar to E. coli in present study. It was $100 \%$ sensitive to aminoglycoside, nitrofurointoin, imipenam. With minimal resistance to cephalosporin (cefotaxim) and maximum resistance to ampicillin and cotrimaxazole. Findings from other studies of Bryce et al., (2016), Salles et al., (2013) support above findings.

Enterococci species were the second most common uropathogens in the present study and most common gram positive organism. Enterococci were $100 \%$ susceptible to vancomycin, ticoplanin and nitrofurintoin and to lesser degree to erythromycin (74\%), doxycyclin $(60 \%)$. Similar sensitivity pattern is seen in literature (Shaikh et al., 2008). However Gupta et al., a study from north India has isolated vancomycin resistant enterococci which is a worrisome situation. Other less common pathogens found in present study were Staphylococcus species 21(11\%) Pseudomonas species 11(6\%), and Proteus species 6(3\%). Their prevalence is variable in other studies as well (Foxman, 2014; Ismaili et al., 2011).

Urinary tract infection is one of the commonest infection seen in infants worldwide (Bryce et $a l ., 2016)$. In infants with suspected UTI, the most common strategy is to treat empirically. In developing countries like India majority of patients present to primary care physicians or pediatric clinics where facilities of microbiological testing of urine are not available and empirical treatment is the choice. This has shown to increase drug resistance in uropathogens.

The study conducted by us and similar studies from other part of country will help to formulate antibiotic strategies.

Urinary tract infection is common in infants and it varies with age and gender. Appropriate evaluation of infants with history, physical examination, urine analysis and microbiological testing of urine should be mandatory. This study highlights the better efficacy of nitrofurantoin and aminoglycosides, imipenam and can be included as empirical therapy. However each institution should have bacteriological profile and list of sensitive antibiotics to avoid development of drug resistance.

\section{References}

Bryce A, Hay AD, Lane IF, Thornton HV, Wootton M, Costelloe C. 2016. Global prevalence of antibiotic resistance in paediatric urinary tract infections caused by Escherichia coli and association with routine use of antibiotics in primary care: systematic review and meta-analysis. BMJ., 352:1939.21.

Budd J, Durham A, Gwise T.2013. Measurement procedure comparison and bias estimation using patient samples: approved guideline. CLSI document EP09-A3. Wayne (PA): Clinical and Laboratory Standards Institute.

Butler C, O'Brien K, Pickles T, Hood K, Wootton M, Howe R. 2015.Childhood urinary tract infection in primary care: a prospective observational study of prevalence, diagnosis, treatment, and recovery. Br J Gen Prac., 65(633):7.

Chang SL, Shortliffe LD. Pediatric urinary tract infections.2006. Pediatr Clin North Am., 53(3):379-400.

Downs SM.1999. Technical report: urinary tract infections in febrile infants and young children. The Urinary Tract Subcommittee of the American Academy of Pediatrics Committee on Quality Improvement. Pediatrics., 103:e54.

Elder JS. Urinary tract infections. In: Kliegman RM, Behrman RE, Jenson HB, Stanton BE, editors.2007. Nelson Textbook of Pediatrics. Philadelphia: Saunders. 22238.

Foxman B.2014. Urinary tract infection syndromes: occurrence, recurrence, bacteriology, risk factors, and disease burden. Infect Dis Clin North Am., 28:1--13.2 
Gould CV, Umscheid, CA, Agarwal RK, Kuntz G, Pegues DAand the Healthcare Infection Control Practices Advisory Committee (HICPAC).2009. Guideline for prevention of catheter associated urinary tract infections. Department of Health and Human Services, USA and Center for Disease Control and Prevention (CDC\&P). Available from: http://www.cdc.gov/ncidod/dhqp/gl_cathe ter_assoc.html

Gupta P, Mandal J, Krishnamurthy S, Barathi D, Pandit N.2015. Profile of urinary tract infections in paediatric patients. Indian $J$ Med Res., (4) 2015, 473-477

Ismaili K, Wissing KM, Lolin K, Le PQ, Christophe C.2011. Characteristics of first urinary tract infection with fever in children: a prospective clinical and imaging study. Pediatr Infect Dis J., 30(5): 371-374

Jitendranath A, Radhika R, Bhargavi L, Bhai G, Beevi R. 2015. Microbiological Profile of Urinary Tract Infection in Pediatric Population from a Tertiary Care Hospital in South Kerala. J Bacteriol Mycol Open Access, 1(1): 00002

Mori R, Lakhanpaul M, Verrier-Jones K.2007. Diagnosis and management of urinary tract infection in children: summary of NICE guidance. BMJ., 335(7616): 395 \pm 7 .

Patel P, Garala RN.2014. Bacteriological profile and antibiotic susceptibility pattern (antibiogram) of urinary tract infections in paediatric patients. JMDS Vol. 2 (1), 141.

Rai GK, UpretiHC, Rai SK, Shah KP, Shrestha RM.2008. Causative agents of urinary tract infections in children and their antibiotic sensitivity pattern: a hospital based study. Nepal Med Coll J., 10(2): 86-90.

Salles MJ, Zurita J, Mejía C, Villegas MV. 2013. Latin America Working Group on Bacterial Resistance. Resistant gramnegative infections in the outpatient setting in Latin America. Epidemiol. Infect., 141: 2459-72.

Shaikh N, Morone NE, Bost JE. 2008. Prevalence of urinary tract infection in childhood: a meta-analysis. Pediatr Infect Dis J. 27: 302-8.

Spencer JD, Schwaderer A, McHugh K, Hains DS.2010. Pediatric urinary tract infections: an analysis of hospitalizations, charges, and costs in the USA. Pediatr Nephrol., 25(12): 2469-2475.

Taneja N, Rani P, Emmanuel R, Sharma M. 2004. Significance of vancomycin resistant enterococci from urinary specimens at a tertiary care centre in northern India. Indian J Med Res., 119: $72-4$

Vernon SJ, Coulthard MG, Lambert HJ, Keir MJ, Matthews JN. 1997. New renal scarring in children who at age 3 and 4 years had had normal scans with dimercaptosuccinic acid: follow up study. BMJ., 315(7113): 905 \pm 8 .

Winberg J, Andersen H, Bergström T. 1974. Epidemiology of symptomatic urinary tract infection in childhood. Acta Paediatr., 63: 1-20.

Yilmaz Y, Tazegun ZT, Aydin E. 2016. Bacterial uropathogens causing urinary tract infection and their resistance patterns among children in Turkey. Iran Red Crescent Med J., 18: e26610

\section{How to cite this article:}

Neha Shah, Sachin Wankhede and Arvind V. Bhore. 2018. Bacteriological Profile of Urinary Tract Infection in Infants. Int.J.Curr.Microbiol.App.Sci. 7(04): 2486-2491. doi: https://doi.org/10.20546/ijcmas.2018.704.285 